\title{
When a workforce strategy won't work: Critique on current policy direction in England, UK
}

This editorial is based on findings from a regional review of educational commissioning, and workforce issues for one Strategic Health Authority in England. Known as the 'Valued People Project' (VPP) it commenced in May 2008 and was undertaken in response to expressed concerns by regional key stakeholders regarding education commissioning specifically of pre-registration learning disability nursing, and the contribution of the specialist learning disability health workforce more generally (Gates, 2009). This work was also undertaken as a consequence of the scale and cumulative effect of changes to education, workforce, professional regulation and central health and social care policy that has affected people with learning disabilities, and the services and personnel that support them. The overarching aims of this strategic review were to;

- map the range and extent of services and service providers across NHS South Central,

- establish an evidence base that would support a strategic approach to future educational commissioning in learning disability,

- establish how specialist NHS learning disability staff were deployed [with the possibility of the development of a new learning disability practitioner for health and social care],

- articulate a flexible learning and development framework that would support the career framework for staff who work with individuals with learning disabilities,

- develop an educational model that would ensure that all education programmes commissioned by NHS South Central will have incorporated key competencies related to caring for individuals with learning disabilities,

- develop a communication strategy to inform services and practitioners of ongoing work and outcomes.

The project adopted a structured multi-method approach to systematically generate robust evidence based on a number of data sources to inform education commissioning and plan for future workforce requirements. These data sources 
included; postal questionnaire survey of Local Learning Disability Partnership Boards, semi-structured interviews with commissioners of services, focus groups with a wide group of key stakeholders, along with analysis of relevant literature and policy documentation, and desk top research. The project was overseen by an expert strategic steering group and engaged with key stakeholders that included, people with learning disabilities, parents and family carers, service managers from health and social care as well as third sector, learning disability practitioners, and academic staff from Higher Education Institutions, and students from Higher and Further Education Institutions; ensuring that there was the widest possible consultation.

This editorial reports on one aspect of the work undertaken; workforce issues. It will predominantly focus on learning disability nursing, although other members of the interdisciplinary specialist learning disability health team are also briefly considered. It will be shown that, for England at least, that there will be a shortage of learning disability nurses in the short to medium term as a consequence of an ageing workforce aligned with a decline in the numbers of pre-registration learning disability nurses being commissioned, and when this is understood within a context of the numbers of starters and qualifiers on and from programmes of professional preparation this has to be seen as problematic. Simply, stated the dwindling number of learning disability nurses, caused by a combination of factors, in my view will not be sufficient to meet the growing needs of some people with learning disabilities who will need specialist support by the NHS, as well as growing demands placed on this workforce by the third sector, and the wider health and social care economies. This potential shortage will lead to a compromised workforce caused by unprecedented demands made from them, arising from a known increase in the prevalence of learning disabilities particularly that of young people with profound learning disabilities with complex needs who are increasingly surviving into adulthood (Emerson, 2009). In addition there is to be an increase in the older population of people with learning disabilities, as well as increases in prevalence of learning disabilities in some ethnic groups (Emerson, 2009); these known increases will inevitably lead to increased demands from specialist learning disability services. Therefore, it would not seem 
unreasonable to assume that one might expect either maintenance in the numbers of practitioners in this workforce, or a consequential increase. However, when data were analysed for this SHA a number of worrying trends appeared to surface. Figure 1 shows the current age profile of the learning disability specialist workforce (based on FTE) for NHS South Central. If a retirement age of 60 is assumed, then $5.9 \%$ of the workforce has currently reached retirement age, and a further $13.6 \%$ will reach retirement age within the next 5 years. In addition a significant number of this learning disability workforce may be eligible to retire at 55 in this case $19.5 \%$ of the current workforce has already reached retirement age.

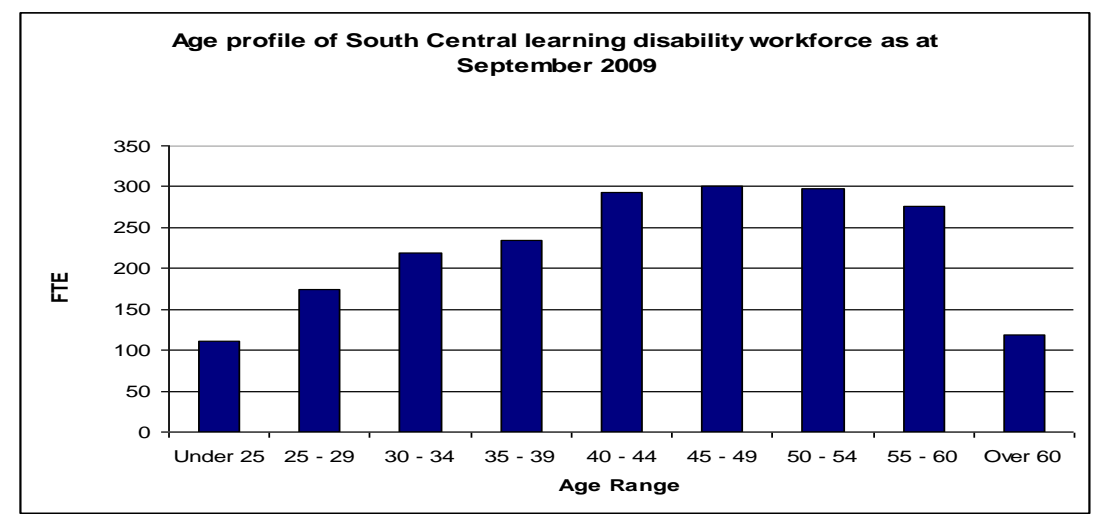

Figure 1 Current age profile of specialist learning disability workforce based on FTE

Now in the case of learning disability nurses Figure 2 shows a reducing supply of learning disability nurses when compared with predicted demand. The demand is based on epidemiological studies showing unprecedented increases in the number of people with learning disabilities along with complexity of health and social care need. For example, a recent study in Sheffield (Parrott, Tilley and Wolstenholme, 2008) has pointed to a $25 \%$ increase in the number of people with a learning disability, and the number of children and young people with profound and multiple learning disabilities has increased by nearly $120 \%$. A more recent and wider epidemiological estimate has been undertaken, and this has calculated that during the period 2009 - 2026 there will be annual increase of $1.8 \%$ in the numbers of adults with profound and multiple learning disabilities. This would mean for an average area in England, with a population of 250,000, that the number of adults with profound and multiple disabilities will increase from 78 in 2009 to 105 in 202 , and that the number of young people becoming adults in any given year will 
rise from 3 in 2009 to $~ 5$ in 2026 (Emerson, 2009). Further it has previously been estimated that between 2001 and 2021 there will be a:

- $10 \%$ increase in the number of adults with learning disabilities known to services.

- $36 \%$ increase in the number of adults with learning disabilities aged $60_{+}$ who are known to services.

- $14 \%$ increase in the total number of adults with learning disabilities in England.

- $38 \%$ increase in the total number of adults with learning disabilities aged 60+ in England.' (Emerson and Hatton, 2004)

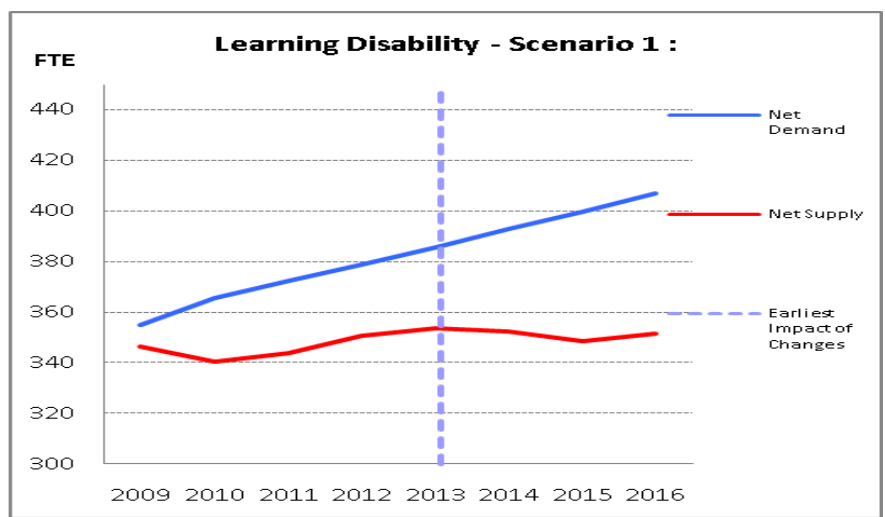

Source: NHS SC CAST Tool 2009/10

Figure 2 Population demands of people with profound and multiple learning disabilities

Thus figure 2 demonstrates data showing a potential shortage in the supply of learning disability nurses - albeit from one Strategic Health Authority. This potential shortage combined with a parallel increase in population demands, a draw from specialist services to support the demands from the wider NHS, and simultaneously a growing demand from the third sector all suggest that there is a need to maintain commissions of pre-registration learning disability nursing, and I do not believe this to be the case; in England at least. It would seem to me that we are heading to an unavoidable learning disability nursing workforce shortfall in the short to medium term unless the NHS and the DOH takes immediate remedial action. As if to accentuate this point a recent publication by the NHS Information Centre: for health and social care (NHS The Information Centre, 2010) gave the following growing vacancy factors for learning disability nursing; 2008 [1.3\%]; 2009 [1.7\%]; and 2010 [2.0\%], and this is for the NHS alone and does not include an 
'unknown' and 'unquantifiable' demand of this workforce from the third sector. And this is within a context of national data that shows a fall in the numbers of learning disability nurses employed by the NHS in the period from 1995 - 2005 from 12,504 to 7,197 , and at least 5 of the 10 SHAs under recruiting to target by between 5$10 \%$ for 2008 . Additionally analysed data from the VPP has demonstrated that for NHS South Central between 2006 -2008 Higher Education Institutions missed their commissions by $\sim 50 \%$, and this was compounded by an additional loss of $\sim 25 \%$ of students through attrition (Gates, 2009).

It is now almost ten years since the government of the time stated as an objective that it wished, to ensure that social and health care staff working with people with learning disabilities are appropriately skilled, trained and qualified; and to promote a better understanding of the needs of people with learning disabilities amongst the wider workforce' (DOH, 2001). And more recently that 'the workforce across public services are given the appropriate support and training to equip them with the values, skills and knowledge to deliver the Valuing People Now priorities' (DOH, 2009).

I would argue, for England at least, whereas recent workforce planning 'toolkits' meant for local workforce planning such as the 'Resource Pack for Learning Disabilities' (NWP, 2007) and the 'Best Practice Guidance for Learning Disability Partnership Boards' (DOH, 2009) are to be welcomed, that they will not sufficiently address the need for strategic influence for this future workforce. This is because local planning almost by definition is necessarily deprived of any regional or national overview, subsequently it is at the very least challenging, and dare one say almost impossible to plan strategically. Also, no account, in either of these documents, is given to the interface between workforce planning and current arrangements for education commissioning in the health sector at least, the latter of which is both bureaucratic often involving complex contractual negotiations with Higher Education providers, and which may well require a three to four year 'roll out' from the commissioning process to that of providing a professionally qualified practitioner. Neither is at all clear as to whether the Local Learning Disability Partnership Boards, who have lead responsibility for workforce planning, have the sufficient expertise and or experience in any of these issues. I also believe that 5 
workforce planning and education commissioning issues are further compounded by inadequate central policy direction, for example, in the latest Valuing People Now (DOH, 2009) policy document, although the social care workforce was specifically identified, the contribution of the specialist NHS learning disability workforce to the lives of people with learning disabilities and their families was notable by its absence. It is clear that strategic planning for a workforce for the future has to be able to accommodate the significance and implications of both the personalisation agenda and personal budgets for health and social care (DOH, 2010) and this makes it imperative that such planning is undertaken as a collaborative enterprise designed to meet the needs of people with learning disabilities and their families, and not political imperatives. This will necessarily involve local, regional and national stakeholders from the wider health and social care economies, crucially involving the third sector, as well as users and commissioners of services. Interestingly a recent publication from Skills for Care provides some useful data concerning the social care workforce for adults with learning disabilities (NMDS - SC briefing issue 10 - adults with learning disabilities, 2009). Their data suggests that this workforce has more men, receive better pay and they are more likely to hold relevant qualifications when compared with NMDS-SC overall workforce data. Establishments are more likely to be from the voluntary sector, and have lower turnover rates although they have higher vacancy rates $4.5 \%$ compared with $2.6 \%$ in services for older people - suggesting that attracting staff into this area of care provision is potentially problematic. Some of the assertions contained within this document however, do require further secondary and independent analysis as original data descriptors are not present, so whereas as an example it talks of this workforce being better qualified - we do not what qualifications these are nor when they were obtained. Regarding the workforce in social care they now have potential access to a Level 2 and 3 Diploma in Health and Social Care that has replaced both the Learning Disability Qualification [and prior to that the Learning Disability Award Framework] and the NVQ in Health and Social Care these staff can pursue the adults with learning disabilities pathway through the Diploma, together with the Level 2 and 3 certificate in supporting people with learning disabilities. And whereas these programmes are to be welcomed it has to be noted that this is third change to 6 
qualifications for this part of the workforce since 2001 and this does beg the question as to their stability and credibility amongst employers of services that support people with learning disabilities. Also if it is to be assumed, quite wrongly in my view, that we no longer need a specialist NHS learning disability workforce and that this workforce can be replaced by a social care workforce then I think people with learning disabilities, their families and employers will need to be reassured that this workforce practices to an ethical code of conduct and therefore this workforce will be accountable for their practice. That there is a suitable development framework for their continuing professional development and perhaps as importantly that the 'general public' will have confidence that this workforce will be deemed to have verifiable competence as opposed to knowledge and in supporting the wide range of some times complex needs of people with learning disabilities.

Regardless of the continued and unnecessary divide between health and social care, and because of a very complex landscape of service delivery and support, we should be drawing on the many talents of all, and that must include the specialist NHS learning disability workforce. I believe that the continuing and 'apparent oversight' and lack of clarity as to the future of a specialist NHS learning disability workforce serves the interests of no one. It will leave this part of the NHS workforce vulnerable and low in morale, and will in the medium to long term deny people with learning disabilities and their family's access to specialist services. A continuing reduction in this workforce will also result in a shortage of specialist practitioners to advise and support the wider health and social care economies to enable to people with learning disabilities to be supported in their chosen life style; in short I think we are moving to period where the national strategy for the whole of the learning disability workforce will simply not work.

Clearly whereas data from the VPP should not necessarily be extrapolated more widely across the other nine SHAs in England there is I believe a need for urgent attention to be paid to workforce planning issues more widely as anecdotal evidence also points to a potential crisis looming. This crisis is not necessarily an 
issue confined to learning disability nursing alone the Valuing People Team at the DOH (Carmichael et al, 2009) has recently commented that;

'in many parts of the country people with learning disabilities and family carers say that access to specialist NHS staff such as speech and language therapists, occupational therapists, clinical psychologists is very limited this should be investigated and the necessary measures taken to ensure this is addressed. The population of people with learning disabilities will increase over the next 20 years and this needs to be factored into all planning arrangements.'

$\mathrm{DOH}$ and the NHS will need to proactively collaborate with all its partners in health and social care to ensure that the NHS specialist learning disability workforce is modernised and skilled, and has sufficient capacity to meet the needs of people with learning disabilities who will undoubtedly continue to seek to use specialist NHS services. Of course none of the arguments put forward in this editorial negates the need for the wider NHS health staff to be adequately prepared to meet the needs of people with learning disabilities in all NHS care settings, and that they are aware of, and are able to make reasonable adjustments for their inclusion, ensuring that their rights are up held, and that they are not discriminated against because of their disability.

To conclude I believe that the increase in prevalence of, and the associated changes to the complexity of need of some people with learning disabilities, and the unprecedented demands that this will make on both health and social care services means that the $\mathrm{DOH}$ and the NHS need to urgently address workforce issues for learning disability nursing as well as the wider specialist NHS learning disability workforce.

Professor Bob Gates,

Academic and Professional Lead - Learning Disabilities,

The University of Hertfordshire, England, UK 


\section{References}

Carmichael, S Poynter, J Mycock, H and Barcham, L (2009) NHS workforce review team - Assessment of Workforce Priorities Summer 2009. Feedback from the Department of Health Valuing People Team, Office of the National Director and National Learning Disability Workforce Group. DOH. London.

Department of Health (2001) Valuing People: a New Strategy for Learning Disability for the 21st Century. Towards Person Centred Approaches: Planning with People. SO: London

Department of Health (2009) Best Practice Guidance for Learning Disability Partnership Boards: Workforce Toolkit. London: Department of Health.

Department of Health (2009) Valuing People: Now. London: Department of Health.

Department of Health (2010) Personalisation through person-centred planning. London: Department of Health.

Emerson, E and Hatton, C (2009) Estimating Future Numbers of Adults with Profound Multiple Learning Disabilities in England. Centre for Disability Research, Lancaster University, UK.

Gates, B (2009) The Valued People Project: Report of a strategic review of educational commissioning and workforce planning in learning disabilities. NHS South Central Strategic Health Authority. Newbury, Berkshire.

National Workforce Projects (2007) People with a learning disability: Workforce planning resource pack. National Workforce Projects. Manchester.

National Health Service: The information centre for health and social care. (2010) NHS vacancies survey England. The Health and Social Care Information Centre.

National Minimum Data Set - Social Care. (2009) Briefing issue 10 - adults with learning disabilities. Skills for Care. London.

Parrott, R Tilley, N and Wolstenholme, J (2008) Changes in demography and demand for services from people with complex needs and profound and multiple learning disabilities. Tizard Learning Disability Review. 13(3). 26-34. 